\title{
Prevalence and Characteristics of Three-rooted Mandibular Molars in Saudi Population: A Retrospective Radiographic Analysis
}

\author{
Ahmed A Alahmed ${ }^{1}$, Rakan M Alabduljabbar ${ }^{2}$, Ziyad M Alrashed ${ }^{3}$, Roshan Uthappa ${ }^{4}$, Toby Thomas ${ }^{5}$, Riyadh Alroomy ${ }^{6}$, \\ Sreekanth K Mallineni ${ }^{7}$
}

\begin{abstract}
Aims: To study the prevalence of three-rooted mandibular permanent first molars (PFM) among Saudi population in Al Zulfi.

Materials and methods: Subjects were selected from the patients' records, College of Dentistry, Majmaah University, Al Zulfi, Kingdom of Saudi Arabia. Healthy patients with the presence of both mandibular PFM, orthopantomogram (OPG) and/or intraoral periapical (IOPA) radiographs were included in the study. All patients included were evaluated by a single examiner using OPG and IOPA radiographs. The occurrence of three roots, status of the tooth and associated anomalies were considered for analysis. Descriptive statistics performed using IBM SPSS (Version 21.0) at a $95 \%$ confidence interval $(p<0.05)$ while the Chi-square test and Fisher test were used for the incidence, and comparison of occurrence of the third root in PFM in the mandibular arch.

Results: A sample of 433 subjects was selected randomly based on our inclusion criteria. Only 24 (5.5\%) subjects had evidence of three-rooted mandibular PFM with the mean age of 27.67 years. The frequency of bilateral and unilateral three-rooted mandibular PFM was $2.3 \%$ (10) and $3.2 \%$ (14), respectively. Overall $41.6 \%$ of subjects were witnessed with the bilateral occurrence and unilateral occurrence was $58.4 \%$ [(57\% (8) were on the right and $42 \%(6)$ were left side] $(p<0.05)$. However, no significant relationship was observed between the left and right occurrence of three-rooted mandibular PFM ( $p<0.05)$. Thirty-four (7.8\%) PFM were presented with three roots and among them, $70 \%(24)$ received treatment. Dilacerations (12.5\%), taurodontism (37\%) and hypodontia (4\%) were associated with three-rooted PFM in the mandibular arch. Kappa statistics showed excellent intra-examiner reliability $(\kappa=0.9)$.

Conclusion: The prevalence of three-rooted PFM was 5.5\% in the present study and unilateral occurrence is very common. Over $70 \%$ of these PFM received dental treatment in Saudi Arabia population. Always, IOPA radiographs are required to confirm the evidence of three-rooted mandibular PFM where three-dimensional imaging is not available.

Clinical significance: An Eagle's eye of an endodontist on three-rooted PFM is of utmost importance in the treatment protocol when the tooth is planned for root canal therapy.

Keywords: Dental anomaly, First permanent molar, Third root.

The Journal of Contemporary Dental Practice (2020): 10.5005/jp-journals-10024-2745
\end{abstract}

\section{INTRODUCTION}

One of the most common purposes of endodontic treatment is to eliminate all microorganisms from the root canal system. The most common failure of endodontic therapy is the inadequate removal of infected pulp tissues from the root canal. ${ }^{1}$ For obtaining better results in endodontic therapy, the clinician needs to have good knowledge about tooth morphology and understand various anatomical variations in the permanent first molars (PFM). Naturally PFM erupt in humans with two roots (mesial and distal root) in mandibular arch, in rare cases PFM erupt with extra root located distolingual and Carabelli was the first one to explore this entity and described this distinct feature in mandibular PFM. ${ }^{2}$ This macrostructure appears in various locations, like if the extra root is located distolingually called as radix entomolaris (RE) whereas if it is located as separated or fused to buccal or mesiobuccally it is termed as radix paramolaris (RP). ${ }^{3}$ A RP can be found mainly on the third mandibular molars, and less frequently seen in the first and second mandibular molars with a frequency of $0.5-1 \%{ }^{4}$ The changes in the normal anatomy of roots in the mandibular molars are identified by very careful radiographic interpretation. The precise diagnosis of the third root is necessary to overcome any complications or missing of the canal during the root canal therapy.

\footnotetext{
${ }^{1-6}$ Department of Restorative Dental Sciences, College of Dentistry, Majmaah University, Al Zulfi, Riyadh, Kingdom of Saudi Arabia

${ }^{7}$ Department of Preventive Dental Sciences, College of Dentistry, Majmaah University, Al Zulfi, Riyadh, Kingdom of Saudi Arabia

Corresponding Author: Roshan Uthappa, Department of Restorative Dental Sciences, College of Dentistry, Majmaah University, Al Zulfi, Riyadh, Kingdom of Saudi Arabia, Phone: +966-538504353, e-mail: r.uthappa@mu.edu.sa

How to cite this article: Alahmed AA, Alabduljabbar RM, Alrashed ZM, et al. Prevalence and Characteristics of Three-rooted Mandibular Molars in Saudi Population: A Retrospective Radiographic Analysis. J Contemp Dent Pract 2020;21(2):197-201.

Source of support: Nil

Conflict of interest: None
}

Interpretation of such specific morphological variation on the distal or mesial root contour or the root canal can hint the existence of a third root and eventually this structure has clinical significance. ${ }^{5}$ There are limited studies to support the presence of additional root in mandibular first molars in the Saudi Arabia population and it is necessary to study this variant structure among Saudi patients, 
because of its clinical importance in endodontic therapy. ${ }^{6}$ Hence, the purpose of the present study was to investigate the prevalence of three-rooted mandibular PFM and its occurrence among Saudi patients in the Al Zulfi region.

\section{Materials and Methods}

This study was carried out in the College of Dentistry, Majmaah University. The Institutional Research Ethics Committee, Majmaah University approved the study prior to the commencement of the study (MUREC-SEP.29/COM-2019/3). A cross-sectional retrospective study included a review of patient records using orthopantomogram (OPG) attended the College of Dentistry, Majmaah University, Al Zulfi, Kingdom of Saudi Arabia. Healthy patients with clear OPG taken during the oral diagnostic examination of age group 11-60 years were included in the study. Patients with medical problems and those having unclear images and below 11 years were excluded from the study. Intraoral periapical radiographs (IOPA) taken in addition to the diagnostic purpose were considered. Orthopantomogram radiograph (70 kV, 10 mA, German, Kodak Care Carestream, 9000) and periapical radiograph ( $60 \mathrm{kV}, 10 \mathrm{~mA}$, German, Kodak) were included for final analysis. The random sample was taken from the patients and evaluated for the prevalence and frequency of three-rooted PFM, and occurrence (unilateral or bilateral) was used for data analysis. All the records were screened by a single examiner. Data entered into Microsoft Excel and analyzed using the statistical package for social sciences (SPSS version 21.0, Illinois, Chicago, USA). Mean \pm SD was reported for quantitative variables like frequencies and percentages were reported for qualitative variables. Chi-squared test and Fisher exact test were applied to observe associations between qualitative variables. A $p$ value of $<0.05$ was considered statistically significant and Kappa statistics used for the evaluation of intra-examiner reliability.

\section{Results}

Randomly 433 subjects were selected for the study. The age ranges from 11 years to 60 years with a mean age of $27.6 \pm 8.6$ years. Only 24 (5.5\%) subjects had evidence of three-rooted mandibular PFM and overall 34 (7.8\%) PFMs were observed with three roots (Fig. 1). The frequency of bilateral and unilateral three-rooted mandibular

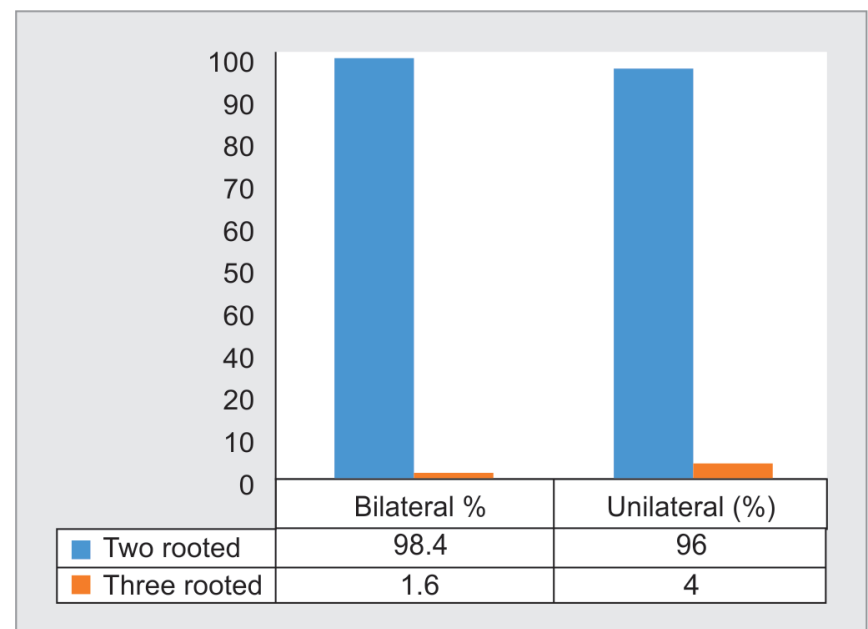

Fig. 1: Descriptive analysis of the three-rooted molars in the Saudi Arabian population
PFM was 2.3\% (10) and 3.2\% (14), respectively (Table 1). Overall $41.6 \%$ (10) of subjects were witnessed with the bilateral occurrence, while unilateral occurrence was $58.4 \%$ (14) [57\% (8) were on the right and $42 \%$ (6) were left side], respectively $(p<0.05)$. Thirty-four PFM were presented with three roots and among them, $70 \%$ (24) were received treatment. Intraoral periapical radiographs were used along with OPG for the diagnosis of three-roots in PFM (Fig. 2) in 50\% of the study sample. Among the study sample three subjects (12.5\%) exhibited dilacerations of the second premolar in the maxillary arch, $37 \%$ (9) subjects were observed with taurodontism (Fig. 3) and one subject (4\%) was noted with hypodontia (Fig. 4). Kappa statistics showed excellent intra-examiner reliability $(\kappa=0.09)$.

\section{Discussion}

To achieve excellent root canal therapy it is very important to appreciate the normal and abnormal distinction of the teeth morphology. ${ }^{4}$ Nevertheless, the anatomical discrepancies of the roots of PFM are not identified by much of general dental practitioners. ${ }^{5}$ One of the diagnostic tools which give an interpretation of the supernumerary structure is IOPA with various angulations. Careful clinical examination of the patient by using a periodontics probe and check of patient's details, review of patient's history in combination with radiographs technique can obtain the best diagnostic information. ${ }^{6}$

Abella et al. ${ }^{7}$ reported that the prevalence of mandibular PFM exhibiting three roots was $14.4 \%$ in the existing literature. However, in the present study, the prevalence of three-rooted PFM was $5.5 \%$ in the mandibular arch. A Taiwanese study ${ }^{8}$ found $5.0 \%$ of threerooted PFM in the mandibular arch in their 277-study sample and an Indian study ${ }^{9}$ reported $5.9 \%$ of three-rooted PFMs in 586 subjects. These findings are almost similar to the present study. However, both studies ${ }^{8,9}$ used only IOPAs for analysis and in the present study OPG was used as a diagnostic tool to locate the third root of PFMs and additionally available IOPAs were used for the confirmation. Moreover, the third root in mandibular PFM is an accidental finding on OPG but it has higher clinical significance if the tooth is planned for root canal therapy.

The extra or third root in the mandibular PFM may present in the same buccolingual plane as of the two roots, and the superimposition of this structure cannot be acknowledged in the conventional radiographs. It has been stated that the conventional radiographs only provide two-dimensional imaging of threedimensional structures. ${ }^{10}$ Usually, an additional radiograph with different angulations is essential to locate an additional tooth or tooth structure. ${ }^{11}$ Interestingly two-dimensional images and three-dimensional images, canal staining, and tooth clearing technique were used in the literature for the identification of three-rooted PFM in the mandibular arch (Table 2)..$^{12-21}$ Among these imaging techniques, cone-beam computed tomography $(C B C T)$ is known to provide three-dimensional images of the teeth and its anatomical structure with high accuracy and without superimposition. ${ }^{21}$ Nonetheless, most recently it has been reported that panoramic radiographs along with intra-oral images may guide

Table 1: Analysis and distribution of roots per tooth

\begin{tabular}{lll}
\hline & \multicolumn{2}{c}{ No. of roots } \\
\cline { 2 - 3 } No. of teeth & 2 roots & 3 roots \\
\hline 433 & 419 & 24 \\
$100 \%$ & $94.5 \%$ & $5.5 \%$ \\
\hline
\end{tabular}



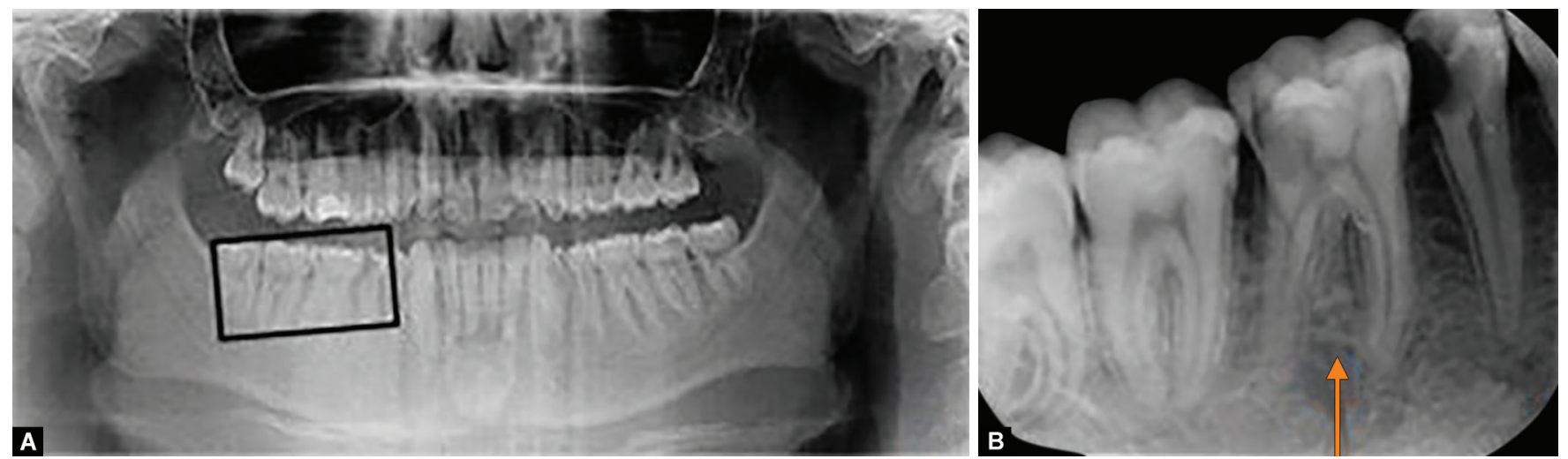

Figs 2A and B: (A) Orthopantomogram; (B) Intraoral periapical radiograph of a patient showing bilateral three-rooted permanent first molars in the mandibular arch

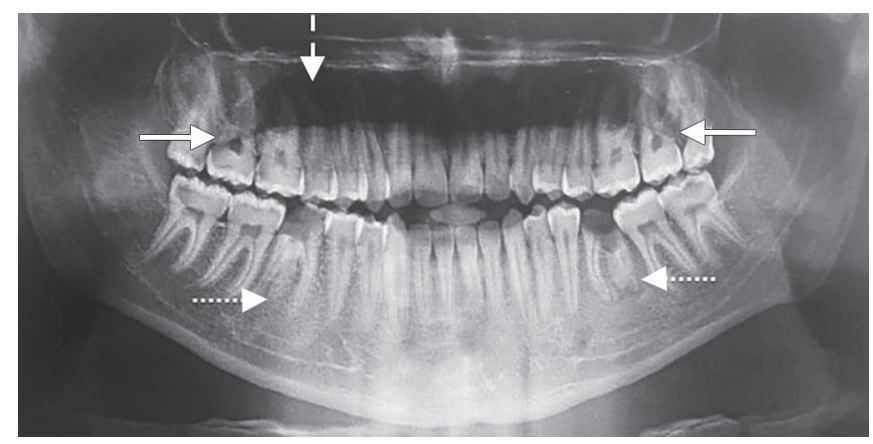

Fig. 3: Orthopantomogram showing dilacerations of tooth 15 root (dashed arrow) and taurodontism in maxillary first permanent molars (arrow) with three-rooted first permanent molar (dotted arrow)

Table 2: Various techniques used for identification of three-rooted molars in mandibular arch

\begin{tabular}{|c|c|c|c|}
\hline Author & Year & Country & Technique \\
\hline Younes et al. ${ }^{12}$ & 1990 & Egypt & $\begin{array}{l}\text { Extracted teeth and } \\
\text { radiographic records }\end{array}$ \\
\hline Al-Nazhan ${ }^{13}$ & 1999 & Saudi Arabia & Clinical records \\
\hline $\begin{array}{l}\text { Gulabivala } \\
\text { et al. }{ }^{14}\end{array}$ & 2001 & Thailand & $\begin{array}{l}\text { Canal staining and } \\
\text { clearing technique }\end{array}$ \\
\hline Peiris et al. ${ }^{15}$ & 2007 & Sri Lanka & Clearing technique \\
\hline Huang et al. ${ }^{16}$ & 2007 & Taiwan & Periapical radiographs \\
\hline Tu et al. ${ }^{17}$ & 2010 & Taiwan & $\begin{array}{l}\text { Vertical bite-wing } \\
\text { radiographs }\end{array}$ \\
\hline Song et al. ${ }^{18}$ & 2010 & Korea & $\begin{array}{l}\text { Computed } \\
\text { tomography }\end{array}$ \\
\hline Tu et al. ${ }^{19}$ & 2009 & Taiwan & $\begin{array}{l}\text { Cone-beam computed } \\
\text { tomography }\end{array}$ \\
\hline Garg et al. ${ }^{20}$ & 2013 & India & $\begin{array}{l}\text { Spiral computed } \\
\text { tomography }\end{array}$ \\
\hline
\end{tabular}

in identification of dental anomalies and development of root. ${ }^{22}$ There was a huge dissimilarity in the prevalence rate of threerooted PFM in CBCT studies. Nonetheless, there was disagreement observed in prior CBCT studies ${ }^{20,23-25}$ used for the identification of three-rooted PFM in the mandibular arch. However, two studies ${ }^{20,23}$ showed the highest occurrence (33.3\% and 29\%) whereas another very low occurrence was noted in the other two studies was $2.6 \%{ }^{24}$ and $2.5 \%,{ }^{25}$ respectively. Furthermore, CBCT is considered an ideal

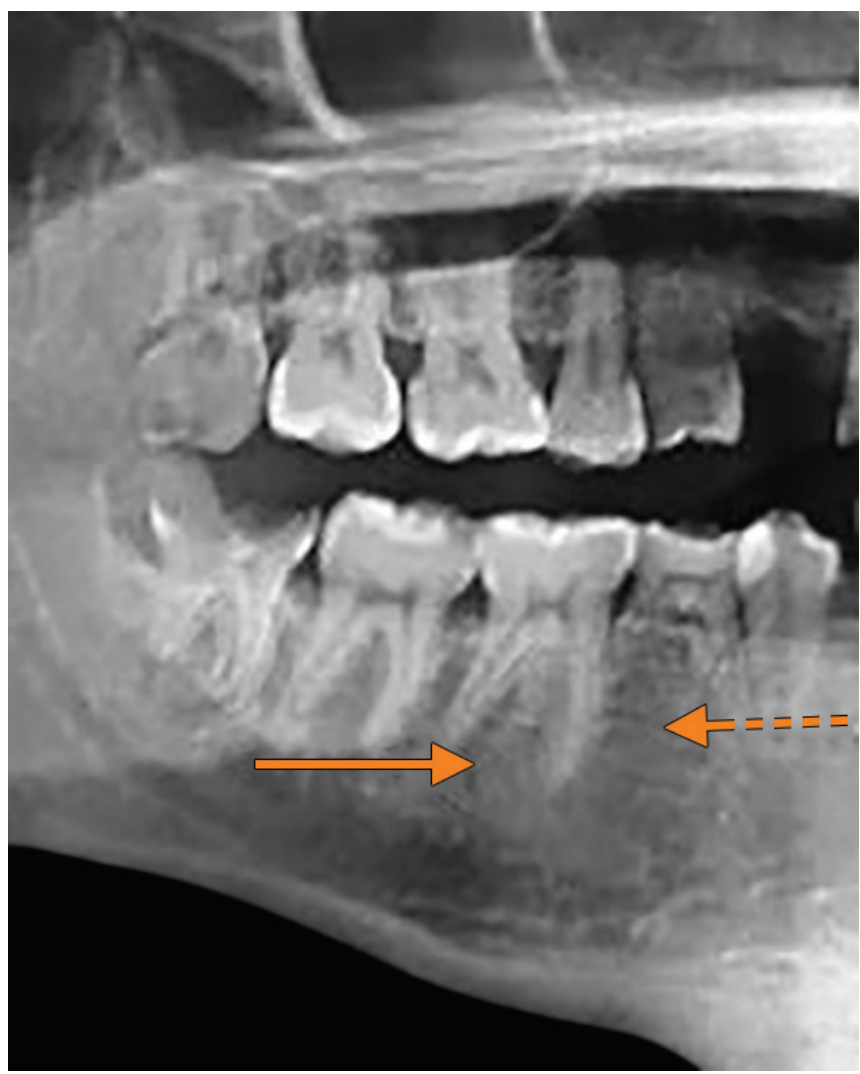

Fig. 4: Cropped panoramic radiograph showing congenitally missing second premolar (dotted arrow) in association with three-rooted first permanent molar (arrow) in mandibular arch

diagnostic imaging modality in the field of modern dental practice including endodontics. ${ }^{21,22}$

Nonetheless, in the present study, the occurrence was found to be $5.5 \%$ (24/433) of all subjects evaluated and 3.9\% (34/866) of all teeth examined while subject and tooth prevalence was $5.5 \%$ and $3.9 \%$, respectively. Published literature revealed that the bilateral occurrence of three roots in PFM in the mandibular arch ranges from $56 \%$ to $68 \% .{ }^{26,27}$ Inversely, a German study reported only unilateral occurrence. ${ }^{28}$ Most recently an Indian study found an incidence of $27.6 \%$ for bilateral occurrence in their study sample. ${ }^{29}$ Surprisingly in the present study the bilateral occurrence of three-rooted PFM was $41.6 \%$ and results were significant with unilateral occurrence. 
Three-rooted Mandibular Molars in Saudi Population

Table 3: Reported details of three-rooted permanent first molars in the mandibular arch in the Saudi population

\begin{tabular}{lllllll}
\hline Author & Year & Sample & $\begin{array}{l}\text { Subject } \\
\text { prevalence (\%) }\end{array}$ & $\begin{array}{l}\text { Tooth } \\
\text { prevalence (\%) }\end{array}$ & Sample details & Technique used \\
\hline $\begin{array}{l}\text { Younes et al. } \\
\text { Al-Nazhan }\end{array}$ & 1990 & 385 & - & 2.33 & Extracted teeth & Clinical records \\
$\begin{array}{l}\text { Bahammam and } \\
\text { Bahammam }\end{array}$ & 1999 & 251 & - & 5.97 & Root canal treated teeth & IOPA \\
$\begin{array}{l}\text { Patil et al. }{ }^{24} \\
\text { Present study }\end{array}$ & 2011 & 280 & - & 6.07 & Extracted teeth & Clear technique \\
\hline
\end{tabular}

Our findings are in contrast with previous studies published in the literature. A Taiwanese study ${ }^{8}$ and a Turkish study ${ }^{30}$ found more three-rooted PFMs are on the right side than the left side. In contrast to these findings, a Singapore study ${ }^{31}$ reported a high prevalence rate on the left side. In the present study right side occurrence was more than that of the left side however, the findings were not statistically significant. However, most of the studies $8,15,28,30$ reported more predilections on the right side and few others on the left side. These contradictory results may be due to the variations in case selection, methods used for detection, and sample size.

Younes et al. ${ }^{12}$ reported that $2.33 \%$ of Saudi subjects with three roots using extracted teeth and radiographic techniques. On the other hand, AL-Nazhan ${ }^{13}$ investigated 251 patients in the college of dentistry of King Saud University in Riyadh using clinical evaluation, and the author found $5.97 \%$ of subjects with three-rooted mandibular PFM. Similarly, Bahammam and Bahammam ${ }^{31}$ found $6.07 \%$ out of 280 extracted teeth of samples with three-rooted mandibular first molars and they used the visual field for screening, this study carried out in King Abdelaziz University in Jeddah. A most recent study done in a College of Dentistry, Jouf University found 3.27\% with three-rooted mandibular first molars and they used CBCT scans. ${ }^{24}$ Among these previous studies (Table 3) ${ }^{12,13,24,31}$ performed in Saudi Arabian populations, two studies were reported with the same prevalence figures and other studies with fewer occurrences than the present study. Interestingly three of our subjects (12.5\%) showed dilacerations of upper right second premolar, taurodontism of maxillary first permanent molars in $37 \%$ (9) subjects and one subject was noted with hypodontia of second premolar. Nevertheless, early description on these dental anomalies in association with threerooted PFM not been discussed in the literature. Moreover, these findings may be coincidental and further studies are recommended to evaluate the relation among this concomitant occurrence. Based on our study result, the prevalence of three rooted is high comparing with other Saudi studies. Therefore, the oral health practitioner must be through with professional and scientific experience in the ways of detection of three-rooted PFMs. Radiographs are the key to confirm the diagnosis for achieving successful root canal therapy and minimize root canal failure.

\section{LiMITATIONS}

The sample size included in the present study was not convenient and the study was performed in the patients attending a teaching hospital for dental treatments, not in the entire population, hence, the study results cannot be generalized.

\section{ConCLUSION}

The three-rooted PFM was 5.5\% subject and 7.8 tooth prevalence in the present study, and unilateral occurrence is very common than the bilateral occurrence. Over $70 \%$ of these PFM received dental treatment in the Saudi Arabia population. Three-rooted PFM may be associated with dilacerations and hypodontia of second premolars, and taurodontism maxillary PFM. Always, IOPA radiographs are required to confirm the evidence of three-rooted mandibular PFM where three-dimensional imaging is not available.

\section{Clinical Significance}

An eagle's eye of an endodontist on three-rooted PFM is of utmost importance in treatment protocol when the tooth is planned for endodontic therapy. Most of the times third root is an accidental finding on the intra-oral periapical radiographs, and always an OPG is clinician first choice of radiograph for the comprehensive examination of the patient. However, in the present study the use of two-dimensional radiographs (OPG and IOPA) in the identification of third or extra root in PFM has been highlighted.

\section{Acknowledgments}

We like to thank our Dean Dr Abdulrahman A Al-Atram, Vice Dean Academics Dr Sami Aldhuwayhi, College of Dentistry, Majmaah University, Saudi Arabia, for their cooperation and support extended to this study. Dr Waqas Sami (PhD Biostatistics) for his assistance with statistical analyses.

\section{References}

1. Cohen AS, Brown DC. Orofacial dental pain emergencies: endodontic diagnoses and management. In: Cohen S, Burns RC. Pathways of the Pulp, 8th ed. Boston, MA, USA: Mosby; 2002. pp. 31-75.

2. Carabelli G. Systematisches Handbuch der Zahnheilkunde, 2nd ed., Vienna: Braumuller and Seidel; 1844. p. 114.

3. Bolk L. Bemerküngen über Wurzelvariationen am menschlichen unteren Molaren. Z Morphol Anthropol 1915;17:605-610.

4. Segura-Egea JJ, Jiménez-Pinzón A, Ríos-Santos JV. Endodontic therapy in a 3-rooted mandibular first molar the: importance of a thorough radiographic examination. J Can Dent Assoc 2002;68(9):541-544.

5. Slaus G, Bottenberg P. A survey of endodontic practice amongst Flemish dentists. Int Endod J 2002;35(9):759-767. DOI: 10.1046/j.13652591.2002.00564.x.

6. Jin GC, Lee SJ, Roh BD. Anatomical study of C-shaped canals in mandibular second molars by analysis of computed tomography. J Endod 2006;32(1):10-13. DOI: 10.1016/j.joen.2005.10.007.

7. Abella F, Patel S, Durán-Sindreu F, et al. Mandibular first molars with disto-lingual roots: review and clinical management. Int Endod J 2012;45(11):963-978. DOI: 10.1111/j.1365-2591.2012.02075.x.

8. Tu MG, Tsai CC, Jou MJ, et al. Prevalence of three-rooted mandibular first molars among Taiwanese individuals. J Endod 2007;33(10): 1163-1166. DOI: 10.1016/j.joen.2007.07.020.

9. Garg AK, Tewari RK, Kumar A, et al. Prevalence of three-rooted mandibular permanent first molars among the Indian Population. J Endod 2010;36(8):1302-1306. DOI: 10.1016/j.joen.2010.04.019.

10. Anthonappa RP, King NM, Rabie AB, et al. Reliability of panoramic radiographs for identifying supernumerary teeth in children. Int J Paediatr Dent 2012;22(1):37-43. DOI: 10.1111/j.1365-263X.2011.01155.x. 
11. Mallineni SK, Anthonappa RP, King NM. Reliability of horizontal and vertical tube shift techniques in the localisation of supernumerary teeth. Eur Arch Paediatr Dent 2016;17(6):455-460. DOI: 10.1007/ s40368-016-0253-9.

12. Younes SA, Al-Shammery AR, El-Angbawi AF. Threrooteded permanent mandibular first molarof Asianan and black groups in the Middle East. Oral Surg Oral Med Oral Pathol 1990;69(1):102-105. DOI: 10.1016/0030-4220(90)90276-X.

13. Al-Nazhan S. Incidence of four canals in root-canal treated mandibular first molars in a Saudi Arabian subpopulation. Int Endod J 1999;32(1):49-52. DOI: 10.1046/j.1365-2591.1999. 00188.x.

14. Gulabivala K, Aung TH, Alavi A, et al. Root and canal morphology of Burmese mandibular molars. Int Endod J 2001;34(5):359-370. DOI: 10.1046/j.1365-2591.2001.00399.x.

15. Peiris HR, Pitakotuwage TN, Takahashi M, et al. Root canal morphology of mandibular permanent molars at different ages. Int Endod J 2008;41(10):828-835. DOI: 10.1111/j.1365-2591.2008. 01428.x.

16. Huang RY, Lin CD, Lee MS, et al. Mandibular disto-lingual root: a consideration in periodontal therapy. J Periodontol 2007;78(8): 1485-1490. DOI: 10.1902/jop.2007.060419.

17. Tu MG, Liu JF, Dai PW, et al. Prevalence of three-rooted primary mandibular first molars in Taiwan. J Formos Med Assoc 2010;109(1): 69-74. DOI: 10.1016/S0929-6646(10)60023-X.

18. Song JS, Choi HJ, Jung IY, et al. The prevalence and morphologic classification of distolingual roots in the mandibular molars in a Korean population. J Endod 2010;36(4):653-657. DOI: 10.1016/ j.joen.2009.10.007.

19. Tu MG, Huang HL, Hsue SS, et al. Detection of permanent threerooted mandibular first molars by cone-beam computed tomography imaging in Taiwanese individuals. J Endod 2009;35(4):503-507. DOI: 10.1016/j.joen.2008.12.013.

20. Garg AK, Tewari RK, Agrawal N. Prevalence of three-rooted mandibular first molars among Indians using SCT. Int J Dent 2013;2013:183869. DOI: 10.1155/2013/183869.
21. Shemesh A, Levin A, Katzenell V, et al. Prevalence of 3-and 4-rooted first and second mandibular molars in the Israeli population. J Endod 2015;41(3):338-342. DOI: 10.1016/j.joen.2014.11.006.

22. Mallineni SK, Jayaraman J, Wong HM, et al. Dental development in children with supernumerary teeth in the anterior region of maxilla. Clin Oral Investig 2019;23(7):2987-2994. DOI: 10.1007/s00784-018-2709-2.

23. Zhang $\mathrm{R}$, Wang $\mathrm{H}$, Tian $\mathrm{YY}$, et al. Use of cone-beam computed tomography to evaluate root and canal morphology of mandibular molars in Chinese individuals. Int Endod J 2011;44(11):990-999. DOI: 10.1111/j.1365-2591.2011.01904.x.

24. Patil SR, Maragathavalli G, Araki K, et al. Three-rooted mandibular first molarsina Saudi Arabian population:aCBCT study. Pesq Bras Odontoped Clin Integr 2018;18(1):e4133. DOI: 10.4034/PBOCI.2018.181.87.

25. Rodrigues $C T$, de Oliveira-Santos C, Bernardineli N, et al. Prevalence and morphometric analysis of three rooted mandibular first molars in a Brazilian subpopulation. J Appl Oral Sci 2016;24(5):535-542. DOI: 10.1590/1678-775720150511.

26. Steelman R. Incidence of an accessory distal root on mandibular first permanent molars in Hispanic children. ASDC J Dent Child 1986;53(2):122-123.

27. Chandra SS, Chandra S, Shankar P, et al. Prevalence of radix entomolaris in mandibular permanent first molars: a study in a South Indian population. Oral Surg Oral Med Oral Pathol Oral Radiol Endod 2011;112(3):e77-e82. DOI: 10.1016/j.tripleo.2011.02.016.

28. Schäfer $E$, Breuer $D$, Janzen $S$. The prevalence of three-rooted mandibular permanent first molars in a German population. J Endod 2009;35(2):202-205. DOI: 10.1016/j.joen.2008.11.010.

29. Colak H, Ozcan E, Hamidi MM. Prevalence of three-rooted mandibular permanent first molars among the Turkish population. Niger J Clin Pract 2012;15(3):306-310. DOI: 10.4103/1119-3077.100627.

30. Loh HS. Incidence and features of three-rooted permanent mandibular molars. Aust Dent J 1990;35(5):434-437. DOI: 10.1111/ j.1834-7819.1990.tb05426.x.

31. Bahammam LA, Bahammam HA. The incidence of radix entomolaris in mandibular first permanent molars in a Saudi Arabian subpopulation. JKAU Med Sci 2011;18(4):83-90. DOI: 10.4197/Med.18-4.7. 